\title{
To Validate the Role of Electromagnetic and Strong Gravitational Constants via the Strong Elementary Charge
}

\author{
U. V. S. Seshavatharam ${ }^{1, *}$, S. Lakshminarayana ${ }^{2}$ \\ ${ }^{1}$ Honorary Faculty, Institute of Scientific Research on Vedas(I-SERVE), India \\ ${ }^{2}$ Department of Nuclear Physics, Andhra University, India
}

Copyright (C) 2015 by authors, all rights reserved. Authors agree that this article remains permanently open access under the terms of the Creative Commons Attribution License 4.0 International License

\begin{abstract}
Within the atomic medium, in analogy with gravity and Schwarzschild interaction, atomic phenomena can be understood with large values of gravitational constants. It may be noted that, larger the magnitude of gravitational constant, smaller is the magnitude of the operating force. The key points to be noted are: 1) There exists a strong elementary charge and squared ratio of electromagnetic and strong interaction charges is equal to the strong coupling constant. 2) There exists a gravitational constant associated with strong interaction, $G_{s}=$ $3.329561213 \times 10^{28} \quad \mathrm{~m}^{3} \mathrm{~kg}^{-1} \mathrm{sec}^{-2}$ 3) There exists a gravitational constant associated with electromagnetic interaction, $G_{e}=2.374335685 \times 10^{37} \mathrm{~m}^{3} \mathrm{~kg}^{-1} \mathrm{sec}^{-2}$; As the magnitude of operating force is far less than the magnitude of $\left(c^{4} / G\right)$, protons and electrons cannot be considered as black holes. With further research and analysis, massive origin of protons and electrons can be understood. In this paper, by quantifying the strong interaction elementary charge, an attempt is made to validate the role of the proposed electromagnetic and strong interaction gravitational constants.
\end{abstract}

Keywords Schwarzschild's Interaction, Electromagnetic Gravitational Constant, Strong Interaction Gravitational Constant and Strong Elementary Charge

\section{Introduction}

In order to unify cosmology, quantum mechanics and the four observed fundamental cosmological interactions, a 'unified force' is required. In this connection $\left(c^{4} / G\right)$ can be considered as the classical force or astrophysical force limit. For a detailed description on this characteristic limiting force, readers are strongly encouraged to see the historical paper by G.W.Gibbons [1]. Similarly, $\left(c^{5} / G\right)$ can be considered as the classical power limit. If it is true that $c$ and $G$ are fundamental physical constants, then $\left(c^{4} / G\right)$ and $\left(c^{5} / G\right)$ can also be considered as fundamental compound physical constants. These classical limits are more powerful than the Uncertainty limit. These two characteristic limits are for future experimental verification with nuclear weapons, particle accelerators, nuclear reactors and rocket propulsion units etc. Moreover, these two characteristic limits can be understood with future astrophysical and cosmological interpretations, observations and inferences. In contrast to the current notion of black hole physics, the Schwarzschild radius of a black hole [2,3] can be understood with the characteristic astrophysical limiting force of magnitude $\left(c^{4} / G\right)$. Note that by considering $\left(c^{4} / G\right)$, the famous Planck mass can be obtained very easily.

For $\left(c^{4} / G\right)$, we have the following applications:

a) Magnitude of force of attraction or repulsion between any two charged particles never exceeds $\left(c^{4} / G\right)$.

b) Magnitude of gravitational force of attraction between any two massive bodies never exceeds $\left(c^{4} / G\right)$.

c) Magnitude of mechanical force on a revolving/rotating body never exceeds $\left(c^{4} / G\right)$.

d) Magnitude of electromagnetic force on a revolving body never exceeds $\left(c^{4} / G\right)$.

For $\left(c^{5} / G\right)$, we have the following applications:

a) Mechanical power never exceeds $\left(c^{5} / G\right)$

b) Electromagnetic power never exceeds $\left(c^{5} / G\right)$

c) Thermal radiation power never exceeds $\left(c^{5} / G\right)$

d) Gravitational radiation power never exceeds $\left(c^{5} / G\right)$

Proceeding further, it may be noted that, from gravity point of view, so far no model succeeded in understanding the link between strongly interacting massive fermions and massive celestial objects. The authors would like to stress the 
fact, strongly interacting massive fermions are only playing a major role in the formation of observable luminous and non-luminous massive celestial objects that follow gravitational interaction. By interconnecting the strong coupling constant and gravitational constant via the Schwarzschild interaction, in this paper, qualitatively and quantitatively the authors reviewed the basics of strong nuclear interaction along with electron-proton interaction. The authors humbly and sincerely agree that, even though the proposed results are interesting and important at fundamental, they require deep mathematical and theoretical back up to proceed further. Considering the failure of current theoretical models in view - the authors request the science community to recommend the content of this paper for further research and development.

In the recently published papers and references therein $[4,5,6,7]$, by introducing two different gravitational constants (one for the electromagnetic interaction and another for the strong interaction), the authors developed many characteristic unified relations. In this paper, by quantifying the strong interaction elementary charge, an attempt is made to validate the role of the proposed electromagnetic and strong interaction gravitational constants. With further research and analysis, status of their authentic physical existence can be understood.

\section{Understanding the Role of $\left(c^{4} / G\right)$ in Black Hole Formation and Planck Mass Generation}

\subsection{Schwarzschild Radius of a Black Hole}

The most fundamental properties of a black hole are its mass, charge, and angular momentum. Without going too deep into the mathematics of black hole physics, in this section, an attempt is made to understand the Schwarzschild radius of a black hole. In all directions, if a force of magnitude $\left(c^{4} / G\right)$ acts on the mass-energy content of the assumed celestial body, it approaches a minimum radius of $\left(G M / c^{2}\right)$ in the following way. The origin of the force $\left(c^{4} / G\right)$ may be due to self-weight or internal attraction or external compression or something else.

$$
R_{\min } \cong \frac{M c^{2}}{\left(c^{4} / G\right)} \cong \frac{G M}{c^{2}}
$$

If no force (or force of zero magnitude) acts on the mass content $M$ of the assumed massive body, its radius becomes infinity. With reference to the average magnitude of $\left(0, \frac{c^{4}}{G}\right) \cong \frac{c^{4}}{2 G}$, the presently believed Schwarzschild radius can be obtained as

$$
(R)_{\text {ave }} \cong \frac{M c^{2}}{\left(c^{4} / 2 G\right)} \cong \frac{2 G M}{c^{2}}
$$

This proposal is very simple and seems to be different from existing concepts and may be a unified form of Newton's law of gravity, the special theory of relativity and the general theory of relativity.

\subsection{To Derive the Planck Mass}

So far no theoretical model has proposed a derivation for the Planck mass. To derive the Planck mass the following two conditions can be considered.

Assume a gravitational force of attraction between two particles of mass $\left(M_{P}\right)$ separated by a minimum distance $\left(\mathrm{r}_{\min }\right)$ to be,

$$
\left[\frac{G M_{P} M_{P}}{r_{\min }^{2}}\right] \equiv\left(\frac{c^{4}}{G}\right)
$$

With reference to wave mechanics, let

$$
2 \pi \cdot r_{\text {min }} \cong \lambda_{P}=\left[\frac{h}{c \cdot M_{P}}\right]
$$

Here, $\lambda_{P}$ represents the wavelength associated with the Planck mass. With these two assumed conditions, the Planck mass can be obtained as follows.

$$
M_{P}=\sqrt{\frac{h c}{2 \pi G}} \cong \sqrt{\frac{\hbar c}{G}}
$$

\subsection{Understanding the Strength of Any Interaction}

From the above relations it is reasonable to say that,

1) If it is true that $c$ and $G$ are fundamental physical constants, then $\left(c^{4} / G\right)$ can be considered as a fundamental compound constant related to a characteristic limiting force.

2) Black holes are the most compact form of matter.

3) Magnitude of the operating force at the black hole surface is of the order of $\left(c^{4} / G\right)$.

4) Gravitational interaction taking place at black holes can be referred to 'Schwarzschild interaction'.

5) Strength of this 'Schwarzschild interaction' can be assumed to be unity.

6) Strength of any other interaction can be defined as the ratio of operating force magnitude and the classical or astrophysical force magnitude $\left(c^{4} / G\right)$.

7) If one is willing to represent the magnitude of the operating force as a fraction of $\left(c^{4} / G\right)$ i.e. $X$ times of $\left(c^{4} / G\right)$, where $X<<1$, then

$$
\frac{X \text { times of }\left(c^{4} / G\right)}{\left(c^{4} / G\right)} \cong X \rightarrow \text { Effective } G \Rightarrow \frac{G}{X}
$$


If $X$ is very small, $\frac{1}{X}$ becomes very large. In this way, $X$ can be considered as the strength of interaction. Thus, the strength of any interaction is $\frac{1}{X}$ times smaller than the 'Schwarzschild interaction' and effective $G$ becomes $\frac{G}{X}$.

\section{Three Basic Assumptions of Final Unification}

The following three assumptions can be considered in a final unification program $[8,9]$.

Assumption-1: The gravitational constant associated with the electromagnetic interaction, $G_{e} \cong 2.374335685 \times 10^{37} \mathrm{~m}^{3} \mathrm{~kg}^{-1} \mathrm{sec}^{-2}$.

Assumption-2: The gravitational constant associated with the strong interaction,

$G_{s} \cong 3.329561213 \times 10^{28} \mathrm{~m}^{3} \mathrm{~kg}^{-1} \mathrm{sec}^{-2}$.

Assumption-3: There exists strong elementary charge, $e_{s} \cong\left(e_{e} / \sqrt{\alpha_{s}}\right)$ where $e_{s}$ is the assumed strong interaction is elementary charge, $e_{e}$ is the currently believed electromagnetic elementary charge and $\alpha_{s}$ is the currently believed strong coupling constant.

With these three assumptions, the key features of nuclear and atomic structure can be understood. With reference to the Schwarzschild interaction, for electromagnetic interaction, $X \cong 2.8105 \times 10^{-48}$ and for strong interaction, $X \cong 2.004 \times 10^{-39}$. Here the authors would like to stress the fact that, as the magnitude of operating force is far less than the magnitude of $\left(c^{4} / G\right)$, protons and electrons cannot be considered as black holes. Within the nuclear medium, in analogy with gravity and Schwarzschild interaction, nuclear phenomena can be understood with large value of gravitational constant. With further research and analysis, massive origin of protons and electrons can be understood.

\section{Important Results}

A) Strong coupling constant: It can be understood as follows.

$$
\alpha_{s} \cong\left(\frac{e_{e}}{e_{s}}\right)^{2} \cong \frac{G_{e} m_{e}^{3}}{G_{s} m_{p}^{3}}
$$

B) Fine structure ratio: It can be understood as follows.

$$
\alpha \cong \frac{e_{s} e_{e}}{4 \pi \varepsilon_{0} G_{s} m_{p}^{2}}
$$

C) Reduced Planck's constant: It can be understood as follows.

$$
\begin{aligned}
\left.\hbar \cong\left(\frac{e_{e}}{e_{s}}\right)\left(\frac{G_{s} m_{p}^{2}}{c}\right) \cong \sqrt{\alpha_{s}}\left(\frac{G_{s} m_{p}^{2}}{c}\right)\right) & \cong \sqrt{\frac{m_{e}}{m_{p}}} \sqrt{\left(\frac{G_{s} m_{p}^{2}}{c}\right)\left(\frac{G_{e} m_{e}^{2}}{c}\right)} \\
\cong & \frac{\sqrt{\left(G_{s} m_{p}\right)\left(G_{e} m_{e}\right)} * m_{e}}{c} \\
& G_{s} \cong\left(\frac{e_{s}}{e_{e}}\right)\left(\frac{\hbar c}{m_{p}^{2}}\right) \cong\left(\frac{1}{\sqrt{a_{s}}}\right)\left(\frac{\hbar c}{m_{p}^{2}}\right)
\end{aligned}
$$

D) Down and Up quark mass ratio: It can be understood as follows.

$$
\frac{m_{d}}{m_{u}} \cong 2 \pi\left(\frac{e_{e}}{e_{s}}\right) \cong 2 \pi \sqrt{\alpha_{s}}
$$

E) Magnetic moment of proton: It can be understood as follows.

$$
\mu_{p} \cong \frac{e_{s} \hbar}{2 m_{p}} \cong \frac{G_{s} e_{e} m_{p}}{2 c}
$$

F) Magnetic moment of neutron: It can be understood as follows.

$$
\mu_{n} \cong \frac{e_{s} \hbar}{2 m_{n}}-\frac{e_{e} \hbar}{2 m_{n}} \cong \frac{\hbar}{2 m_{n}}\left(e_{s}-e_{e}\right)
$$

G) Magnetic moment of electron: It can be understood as follows.

$$
\mu_{e} \cong\left(\frac{e_{s}}{m_{p}}\right)\left(\frac{G_{e} m_{e}^{2}}{2 c}\right)
$$

H) Magnetic moment of muon: It can be understood as follows.

$$
\mu_{\mu} \cong\left(\frac{e_{s}}{m_{p}}\right)\left\{\left(\frac{m_{e}}{m_{\mu}}\right)\left(\frac{G_{e} m_{e}^{2}}{2 c}\right)\right\}
$$

I) Nuclear charge radius: It can be understood as follows.

$$
R_{0} \cong \frac{2 G_{s} m_{p}}{c^{2}}
$$

J) Root mean square radius of proton: It can be understood as follows.

$$
R_{p} \cong \frac{\sqrt{2} G_{s} m_{p}}{c^{2}}
$$

K) Ratio of rest mass of proton and electron: It can be understood as follows.

$$
\frac{G_{s} m_{p} m_{e}}{\hbar c} \cong \frac{\hbar c}{G_{e} m_{p}^{2}}
$$


On simplification,

$$
\begin{aligned}
\left(\frac{m_{p}}{m_{e}}\right) & \cong\left(\frac{4 \pi \varepsilon_{0} G_{e} m_{e}^{2}}{e_{e}^{2}}\right) /\left(\frac{4 \pi \varepsilon_{0} G_{s} m_{p}^{2}}{e_{s}^{2}}\right) \\
& \Rightarrow \frac{m_{p}}{m_{e}} \cong\left(\frac{G_{e} e_{s}^{2}}{G_{s} e_{e}^{2}}\right)^{\frac{1}{3}} \\
G_{e} & \cong\left(\frac{e_{e}^{2}}{e_{s}^{2}}\right)\left(\frac{m_{p}^{3}}{m_{e}^{3}}\right) G_{s} \cong \alpha_{s}\left(\frac{m_{p}^{3}}{m_{e}^{3}}\right) G_{s}
\end{aligned}
$$

L) Planck's constant: It can be understood as follows.

$$
h \cong \sqrt{\left(\frac{e_{s}^{2}}{4 \pi \varepsilon_{0} c}\right)\left(\frac{G_{e} m_{e}^{2}}{c}\right)}
$$

From relations (20 and 21),

$$
\begin{gathered}
\left\{\begin{array}{l}
G_{s} \cong 3.329561213 \times 10^{28} \mathrm{~m}^{3} \mathrm{~kg}^{-1} \mathrm{sec}^{-2} \\
G_{e} \cong 2.374335685 \times 10^{37} \mathrm{~m}^{3} \mathrm{~kg}^{-1} \mathrm{sec}^{-2}
\end{array}\right. \\
\left\{\begin{array}{l}
\alpha_{s} \cong 0.1151937072 \\
e_{s} \cong 4.720586603 \times 10^{-19} \mathrm{C}
\end{array}\right. \\
\left\{\begin{array}{l}
R_{0} \cong 1.239290981 \times 10^{-15} \mathrm{~m} \\
R_{p} \cong 0.87631106 \times 10^{-15} \mathrm{~m}
\end{array}\right. \\
\left\{\begin{array}{l}
\mu_{p} \cong 1.488142 \times 10^{-26} \mathrm{~J} / \text { Tesla } \\
\mu_{n} \cong 9.817104 \times 10^{-27} \mathrm{~J} / \text { Tesla }
\end{array}\right. \\
\left\{\begin{array}{l}
\mu_{e} \cong 9.27009 \times 10^{-24} \mathrm{~J} / \text { Tesla } \\
\mu_{\mu} \cong 4.485159 \times 10^{-26} \mathrm{~J} / \text { Tesla }
\end{array}\right.
\end{gathered}
$$

The very interesting point to be noted here is that, Proton's magnetic moment is associated with its strong elementary charge. Neutron's magnetic moment seems to be the difference of magnetic moment associated with strong interaction and magnetic moment associated with electromagnetic interaction. Considering relations (7) to (21), the authors would like to say that,

A. Along with the strong elementary charge, within the atomic medium there exit two different gravitational constants and their existence is real, not virtual.

B. Whether quantum constants decide the existence of $\left(G_{s}\right.$ and $\left.G_{e}\right)$ or $\left(G_{s}\right.$ and $\left.G_{e}\right)$ will decide the existence of quantum constants - is for future study.

C. It may be noted that, in nature, one can see one proton, two protons, three protons etc. If nuclear mass is discrete, revolving electron can certainly have a discrete angular momentum. With reference to the concept of 'number of protons', discrete nature of $\hbar, 2 \hbar, 3 \hbar, \ldots$ or $\hbar, \sqrt{2} \hbar, \sqrt{3} \hbar, \ldots$ can be understood.

D. Strong elementary charge and strong gravitational constant play a combined role in understanding the nuclear binding. See section -7 .

\section{Proton-neutron Beta Stability Line}

Inside an atomic nucleus, 'beta decay' is a type of radioactive decay in which a proton is transformed into a neutron, or vice versa. This process allows the atom to move closer to the optimal proton-neutron ratio. The important point here is that most naturally occurring isotopes on Earth are beta stable. Beta-decay stable isobars are the set of nuclides which cannot undergo beta decay. A subset of these nuclides are also stable with regards to double beta decay as they have the lowest energy of all nuclides with the same mass number. This set of nuclides is also known as the 'line of beta stability'. The line of beta stability can be defined mathematically by finding the nuclide with the greatest binding energy for a given mass number and can be estimated by the classical semi-empirical mass formula.

The naturally occurring stable mass number connected with the proton number can be expressed as follows [10].

$$
\begin{aligned}
& A_{s} \cong 2 Z+\left\{\left(\frac{G_{s} m_{p} m_{e}}{\hbar c}\right)\right\}(2 Z)^{2} \\
& \left.\cong 2 Z+\left\{\left(\frac{e_{s}}{m_{p}}\right) /\left(\frac{e_{e}}{m_{e}}\right)\right\}(2 Z)^{2}\right\} \\
& \cong 2 Z+\left(0.00642 * Z^{2}\right)
\end{aligned}
$$

Note that,

$$
\begin{aligned}
& \left(\frac{G_{s} m_{p} m_{e}}{\hbar c}\right) \cong \frac{G_{s} m_{p} m_{e}}{\sqrt{\alpha_{s}} G_{s} m_{p}^{2}} \cong\left(\frac{e_{s}}{m_{p}}\right) /\left(\frac{e_{e}}{m_{e}}\right) \\
& \cong \frac{\text { Specific charge of proton }}{\text { Specific charge of electron }}
\end{aligned}
$$

If $Z=92$, obtained $A_{\mathrm{s}} \cong 238.17$ and its actual stable mass number is 238. See Table 1 for the estimated data. Considering even-odd corrections, naturally occurring stable atomic nuclides can also be fitted with this relation. In addition, super heavy stable atomic nuclides can also be predicted. By considering $\left(\frac{\hbar c}{4 G_{s} m_{p} m_{e}}\right) \cong 155.7985$, magic and semi-magic numbers can be fitted [4,5]. One very interesting observation is that,

$$
\ln \sqrt{\frac{\hbar c}{4 G_{s} m_{p} m_{e}}} \cong \frac{\left(m_{n}-m_{p}\right) c^{2}}{m_{e} c^{2}}
$$

Where $m_{n}, m_{p}$ represent neutron and proton rest masses, respectively. See columns 1 and 2 of table-1. 


\section{To Fit and Understand the Nuclear Binding Energy}

\section{Step-1: To Find the Characteristic Binding Energy Potential}

Individual self potential energy of the strongly interacting proton can be fitted as follows.

$$
\left.\begin{array}{l}
E_{p o t} \cong-\frac{3}{5}\left(\frac{e_{s}^{2}}{4 \pi \varepsilon_{0} R_{p}}\right) \\
\cong-\frac{3}{5}\left(\frac{e_{s}^{2}}{4 \pi \varepsilon_{0}\left(\sqrt{2} G_{s} m_{p} / c^{2}\right)}\right) \cong-8.56 \mathrm{MeV}
\end{array}\right\}
$$

For the semi-empirical mass formula, starting from $Z=30$, at the stable mass numbers it is possible to show that, the ratio of binding energy and proton number is close to 19.7 $\mathrm{MeV}$ and is independent of the stable mass number. See the last column of table-1.

Starting from $Z=30$ to $Z=100$, average value of binding energy/proton number for 71 isotopes is $19.7 \mathrm{MeV}$. Here the authors would like to call this as "nuclear binding energy potential". This energy unit can be fitted as follows.

$$
\begin{aligned}
& B_{0} \cong-\frac{e_{s}^{2}}{4 \pi \varepsilon_{0}\left(G_{s} m_{p} / c^{2}\right)} \cong-\frac{e_{s}^{2} c^{2}}{4 \pi \varepsilon_{0} G_{s} m_{p}} \\
& \cong-20.1734 \mathrm{MeV}
\end{aligned}
$$

In the following sub sections, to fit the nuclear binding the authors consider a value of $19.7 \mathrm{MeV}$ that is very close to $20.17 \mathrm{MeV}$.

\section{Step-2: To Find the Binding Energy at Stable Mass Number of $Z \geq 30$}

For $Z=30$ onwards, at the stable mass number, nuclear binding energy can be approximately fitted with the following relation.

$$
(B)_{A_{s}} \cong-Z * B_{0} \cong-Z * 19.7 \mathrm{MeV}
$$

Step-3: To Find the Binding Energy above and below the Stable Mass Number of $\mathbf{Z} \geq \mathbf{3 0}$

For $Z=30$ onwards, above and below the stable mass number,

$$
(B)_{A} \cong-\left(\frac{A}{A_{s}}\right)^{p}(B)_{A_{s}} \cong-\left(\frac{A}{A_{s}}\right)^{p} Z * 19.7 \mathrm{MeV}
$$

where $p \cong 4 / 3$ if $\mathrm{A}<A_{s}$ and $p \cong 2 / 3$ if $\mathrm{A}>A_{s}$.

\section{Step-4: To Find the Binding Energy at the Stable Mass Numbers of $Z<\mathbf{3 0}$}

For $\mathbf{Z}<\mathbf{3 0}$, at the stable mass number, nuclear binding energy can be approximately fitted with the following relation.

$$
(B)_{A_{s}} \cong-k_{z} * Z * 19.70 \mathrm{MeV}
$$

where $k_{z} \cong\left(\frac{Z}{30}\right)^{\frac{1}{6}}$

\section{Step-5: To Find the Binding Energy above and below the Stable Mass Numbers of $\mathbf{Z}<\mathbf{3 0}$}

For $\mathrm{Z}<30$, above and below the stable mass number, nuclear binding energy can be approximately fitted with the following relation.

$$
\left.\begin{array}{l}
(B)_{A} \cong-\left(\frac{A}{A_{S}}\right)^{p}(B)_{A_{s}} \\
\cong-k_{z}\left(\frac{A}{A_{S}}\right)^{p} Z^{*} 19.70 \mathrm{MeV}
\end{array}\right\}
$$

where $k_{z} \cong\left(\frac{Z}{30}\right)^{\frac{1}{6}}, \quad p \cong 4 / 3$ if $\mathrm{A}<A_{s}$ and $p \cong 2 / 3$ if $\mathrm{A}>A_{s}$.

\section{Step-6: To See the Following Tables and Figures}

In the following table-1 and figure-1, the authors tried to compare the estimated binding energy with data obtained from the semi empirical mass formula (SEMF). In the figures, "Green color" thin curve indicates the binding energy estimated with SEMF and "red color" bold curve indicates the estimated binding energy. In a macroscopic approach, starting from $20 \mathrm{MeV}$ to $2000 \mathrm{MeV}$ one cannot find much difference in both of the curves. Relations (24) to (29) need in depth study at fundamental level. 
Table 1. To fit the stable mass numbers and binding energy at stable mass numbers of $Z=2$ to 100

\begin{tabular}{|c|c|c|c|c|c|}
\hline Proton number & $\begin{array}{l}\text { Estimated stable } \\
\text { mass number }\end{array}$ & Coefficient $\mathrm{k}_{\mathrm{z}}$ & $\begin{array}{l}\text { Estimated binding } \\
\text { energy in } \mathrm{MeV}\end{array}$ & $\begin{array}{l}\text { SEMF binding } \\
\text { energy in } \mathrm{MeV}\end{array}$ & $\begin{array}{c}\text { (SEMF binding energy in } \mathrm{MeV}) / \\
\text { Proton number }\end{array}$ \\
\hline 2 & 4 & 0.636773 & 25.1 & 22.0 & 11.0 \\
\hline 3 & 6 & 0.681292 & 40.3 & 26.9 & 9.0 \\
\hline 4 & 8 & 0.714753 & 56.3 & 52.9 & 13.2 \\
\hline 5 & 10 & 0.741836 & 73.1 & 62.3 & 12.5 \\
\hline 6 & 12 & 0.764724 & 90.4 & 87.4 & 14.6 \\
\hline 7 & 14 & 0.784626 & 108.2 & 98.8 & 14.1 \\
\hline 8 & 16 & 0.802284 & 126.4 & 123.2 & 15.4 \\
\hline 9 & 19 & 0.818188 & 145.1 & 148.9 & 16.5 \\
\hline 10 & 21 & 0.832683 & 164.0 & 167.5 & 16.8 \\
\hline 11 & 23 & 0.846016 & 183.3 & 186.1 & 16.9 \\
\hline 12 & 25 & 0.858374 & 202.9 & 204.7 & 17.1 \\
\hline 13 & 27 & 0.869902 & 222.8 & 223.2 & 17.2 \\
\hline 14 & 29 & 0.880713 & 242.9 & 241.6 & 17.3 \\
\hline 15 & 31 & 0.890899 & 263.3 & 260.0 & 17.3 \\
\hline 16 & 34 & 0.900533 & 283.8 & 290.8 & 18.2 \\
\hline 17 & 36 & 0.909678 & 304.7 & 305.1 & 17.9 \\
\hline 18 & 38 & 0.918386 & 325.7 & 327.2 & 18.2 \\
\hline 19 & 40 & 0.926699 & 346.9 & 341.5 & 18.0 \\
\hline 20 & 43 & 0.934655 & 368.3 & 371.6 & 18.6 \\
\hline 21 & 45 & 0.942286 & 389.8 & 389.6 & 18.6 \\
\hline 22 & 47 & 0.949621 & 411.6 & 407.5 & 18.5 \\
\hline 23 & 49 & 0.956682 & 433.5 & 425.2 & 18.5 \\
\hline 24 & 52 & 0.963492 & 455.5 & 454.6 & 18.9 \\
\hline 25 & 54 & 0.97007 & 477.8 & 468.9 & 18.8 \\
\hline 26 & 56 & 0.976432 & 500.1 & 489.6 & 18.8 \\
\hline 27 & 59 & 0.982593 & 522.6 & 515.2 & 19.1 \\
\hline 28 & 61 & 0.988567 & 545.3 & 532.5 & 19.0 \\
\hline 29 & 63 & 0.994366 & 568.1 & 549.7 & 19.0 \\
\hline 30 & 66 & 1 & 591.0 & 577.9 & 19.3 \\
\hline 31 & 68 & 1 & 610.7 & 592.0 & 19.1 \\
\hline 32 & 71 & 1 & 630.4 & 619.8 & 19.4 \\
\hline 33 & 73 & 1 & 650.1 & 636.6 & 19.3 \\
\hline 34 & 75 & 1 & 669.8 & 653.3 & 19.2 \\
\hline 35 & 78 & 1 & 689.5 & 677.9 & 19.4 \\
\hline 36 & 80 & 1 & 709.2 & 697.0 & 19.4 \\
\hline 37 & 83 & 1 & 728.9 & 721.3 & 19.5 \\
\hline 38 & 85 & 1 & 748.6 & 737.6 & 19.4 \\
\hline 39 & 88 & 1 & 768.3 & 761.6 & 19.5 \\
\hline 40 & 90 & 1 & 788.0 & 780.2 & 19.5 \\
\hline 41 & 93 & 1 & 807.7 & 803.9 & 19.6 \\
\hline 42 & 95 & 1 & 827.4 & 819.8 & 19.5 \\
\hline 43 & 98 & 1 & 847.1 & 843.2 & 19.6 \\
\hline 44 & 100 & 1 & 866.8 & 861.2 & 19.6 \\
\hline 45 & 103 & 1 & 886.5 & 884.4 & 19.7 \\
\hline 46 & 106 & 1 & 906.2 & 909.6 & 19.8 \\
\hline 47 & 108 & 1 & 925.9 & 922.7 & 19.6 \\
\hline 48 & 111 & 1 & 945.6 & 947.7 & 19.7 \\
\hline 49 & 113 & 1 & 965.3 & 962.8 & 19.6 \\
\hline 50 & 116 & 1 & 985.0 & 987.5 & 19.8 \\
\hline
\end{tabular}




\begin{tabular}{|c|c|c|c|c|c|}
\hline 51 & 119 & 1 & 1004.7 & 1009.7 & 19.8 \\
\hline 52 & 121 & 1 & 1024.4 & 1024.6 & 19.7 \\
\hline 53 & 124 & 1 & 1044.1 & 1046.5 & 19.7 \\
\hline 54 & 127 & 1 & 1063.8 & 1070.5 & 19.8 \\
\hline 55 & 129 & 1 & 1083.5 & 1085.1 & 19.7 \\
\hline 56 & 132 & 1 & 1103.2 & 1108.7 & 19.8 \\
\hline 57 & 135 & 1 & 1122.9 & 1130.1 & 19.8 \\
\hline 58 & 138 & 1 & 1142.6 & 1153.3 & 19.9 \\
\hline 59 & 140 & 1 & 1162.3 & 1165.6 & 19.8 \\
\hline 60 & 143 & 1 & 1182.0 & 1188.5 & 19.8 \\
\hline 61 & 146 & 1 & 1201.7 & 1209.3 & 19.8 \\
\hline 62 & 149 & 1 & 1221.4 & 1231.9 & 19.9 \\
\hline 63 & 151 & 1 & 1241.1 & 1245.9 & 19.8 \\
\hline 64 & 154 & 1 & 1260.8 & 1268.2 & 19.8 \\
\hline 65 & 157 & 1 & 1280.5 & 1288.4 & 19.8 \\
\hline 66 & 160 & 1 & 1300.2 & 1310.4 & 19.9 \\
\hline 67 & 163 & 1 & 1319.9 & 1330.4 & 19.9 \\
\hline 68 & 166 & 1 & 1339.6 & 1352.0 & 19.9 \\
\hline 69 & 168 & 1 & 1359.3 & 1363.7 & 19.8 \\
\hline 70 & 171 & 1 & 1379.0 & 1385.1 & 19.8 \\
\hline 71 & 174 & 1 & 1398.7 & 1404.5 & 19.8 \\
\hline 72 & 177 & 1 & 1418.4 & 1425.7 & 19.8 \\
\hline 73 & 180 & 1 & 1438.1 & 1444.8 & 19.8 \\
\hline 74 & 183 & 1 & 1457.8 & 1465.7 & 19.8 \\
\hline 75 & 186 & 1 & 1477.5 & 1484.6 & 19.8 \\
\hline 76 & 189 & 1 & 1497.2 & 1505.1 & 19.8 \\
\hline 77 & 192 & 1 & 1516.9 & 1523.7 & 19.8 \\
\hline 78 & 195 & 1 & 1536.6 & 1544.0 & 19.8 \\
\hline 79 & 198 & 1 & 1556.3 & 1562.4 & 19.8 \\
\hline 80 & 201 & 1 & 1576.0 & 1582.3 & 19.8 \\
\hline 81 & 204 & 1 & 1595.7 & 1600.5 & 19.8 \\
\hline 82 & 207 & 1 & 1615.4 & 1620.2 & 19.8 \\
\hline 83 & 210 & 1 & 1635.1 & 1638.1 & 19.7 \\
\hline 84 & 213 & 1 & 1654.8 & 1657.5 & 19.7 \\
\hline 85 & 216 & 1 & 1674.5 & 1675.2 & 19.7 \\
\hline 86 & 219 & 1 & 1694.2 & 1694.3 & 19.7 \\
\hline 87 & 222 & 1 & 1713.9 & 1711.7 & 19.7 \\
\hline 88 & 226 & 1 & 1733.6 & 1737.5 & 19.7 \\
\hline 89 & 229 & 1 & 1753.3 & 1754.6 & 19.7 \\
\hline 90 & 232 & 1 & 1773.0 & 1773.2 & 19.7 \\
\hline 91 & 235 & 1 & 1792.7 & 1790.2 & 19.7 \\
\hline 92 & 238 & 1 & 1812.4 & 1808.5 & 19.7 \\
\hline 93 & 241 & 1 & 1832.1 & 1825.2 & 19.6 \\
\hline 94 & 245 & 1 & 1851.8 & 1848.3 & 19.7 \\
\hline 95 & 248 & 1 & 1871.5 & 1864.8 & 19.6 \\
\hline 96 & 251 & 1 & 1891.2 & 1882.6 & 19.6 \\
\hline 97 & 254 & 1 & 1910.9 & 1898.9 & 19.6 \\
\hline 98 & 257 & 1 & 1930.6 & 1916.5 & 19.6 \\
\hline 99 & 261 & 1 & 1950.3 & 1938.7 & 19.6 \\
\hline 100 & 264 & 1 & 1970.0 & 1956.1 & 19.6 \\
\hline
\end{tabular}




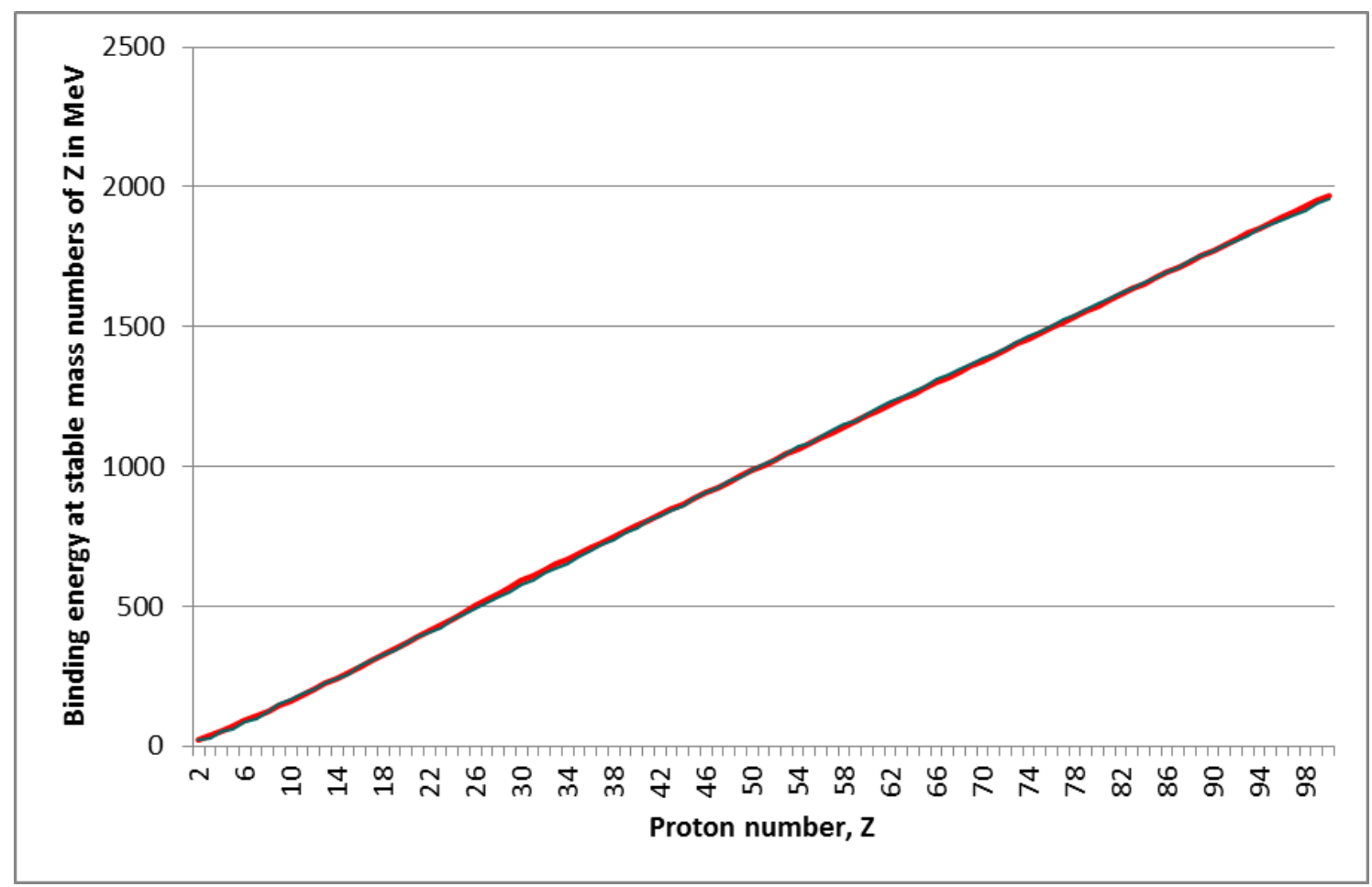

Figure 1. Binding energy at stable mass numbers of $Z=2$ to 100

In the following table- 2 and figure- 2 , the authors tried to compare the estimated binding energy of isotopes of $Z=60$ with data obtained from the semi empirical mass formula (SEMF). In the figure-2, "Green color" curve indicates the binding energy estimated with SEMF and "red color" dashed curve indicates the estimated binding energy.

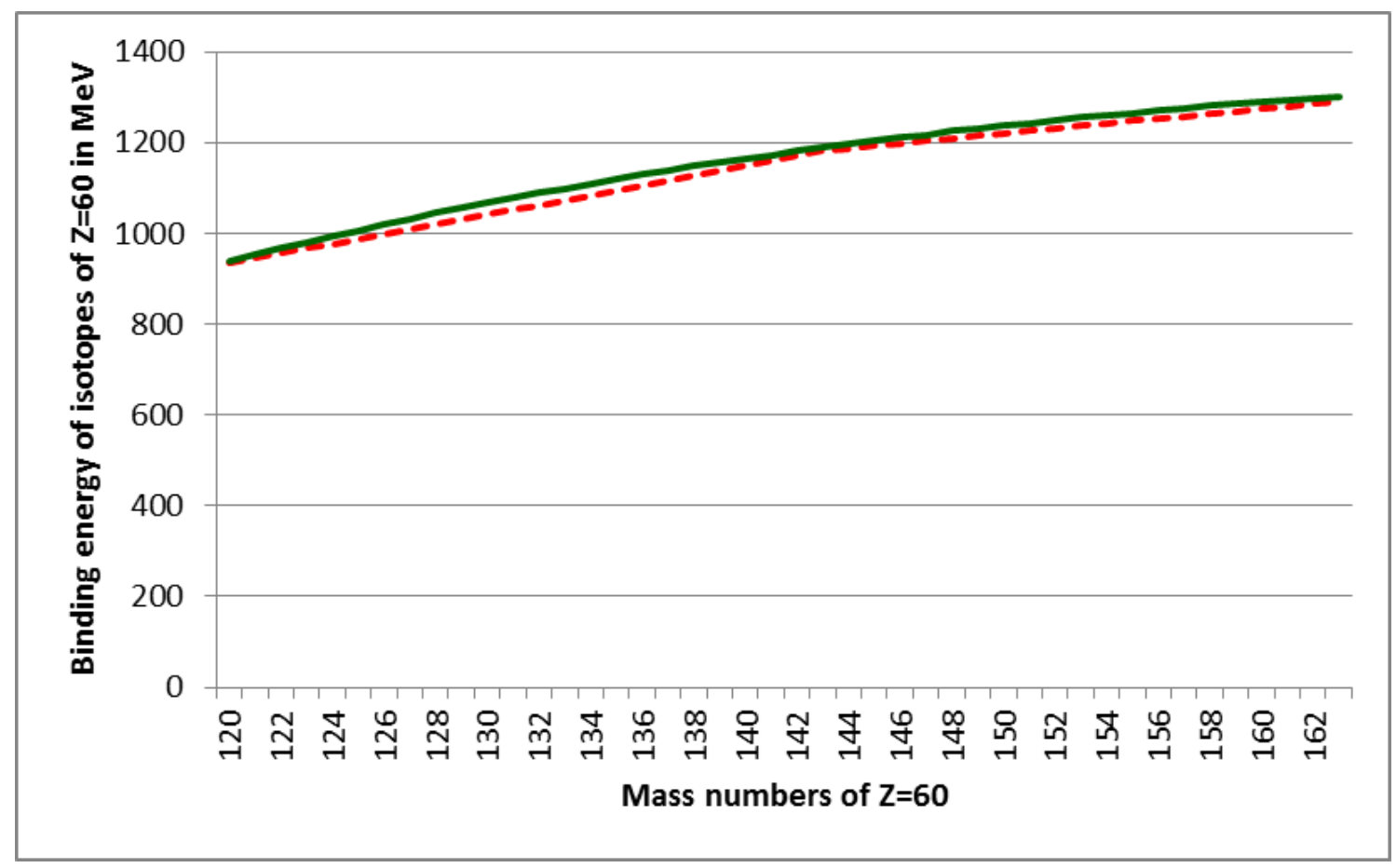

Figure 2. To fit the binding energy of isotopes of $Z=60$ 
To Validate the Role of Electromagnetic and Strong Gravitational Constants via the Strong Elementary Charge

Table 2. To fit the binding energy of isotopes of $Z=60$

\begin{tabular}{|c|c|c|c|c|c|}
\hline Proton number & $\begin{array}{l}\text { Estimated stable } \\
\text { mass number }\end{array}$ & Mass number & Coefficient $\mathrm{k}_{\mathrm{z}}$ & $\begin{array}{l}\text { Estimated binding } \\
\text { energy in } \mathrm{MeV}\end{array}$ & $\begin{array}{l}\text { SEMF binding energy } \\
\text { in } \mathrm{MeV}\end{array}$ \\
\hline 60 & 143 & 120 & 1 & 935.6 & 938.9 \\
\hline 60 & 143 & 121 & 1 & 946.0 & 952.4 \\
\hline 60 & 143 & 122 & 1 & 956.4 & 967.6 \\
\hline 60 & 143 & 123 & 1 & 966.9 & 980.3 \\
\hline 60 & 143 & 124 & 1 & 977.4 & 994.7 \\
\hline 60 & 143 & 125 & 1 & 987.9 & 1006.7 \\
\hline 60 & 143 & 126 & 1 & 998.5 & 1020.4 \\
\hline 60 & 143 & 127 & 1 & 1009.0 & 1031.7 \\
\hline 60 & 143 & 128 & 1 & 1019.7 & 1044.8 \\
\hline 60 & 143 & 129 & 1 & 1030.3 & 1055.4 \\
\hline 60 & 143 & 130 & 1 & 1040.9 & 1067.8 \\
\hline 60 & 143 & 131 & 1 & 1051.6 & 1077.8 \\
\hline 60 & 143 & 132 & 1 & 1062.4 & 1089.6 \\
\hline 60 & 143 & 133 & 1 & 1073.1 & 1099.0 \\
\hline 60 & 143 & 134 & 1 & 1083.9 & 1110.2 \\
\hline 60 & 143 & 135 & 1 & 1094.7 & 1119.0 \\
\hline 60 & 143 & 136 & 1 & 1105.5 & 1129.7 \\
\hline 60 & 143 & 137 & 1 & 1116.3 & 1138.0 \\
\hline 60 & 143 & 138 & 1 & 1127.2 & 1148.0 \\
\hline 60 & 143 & 139 & 1 & 1138.1 & 1155.8 \\
\hline 60 & 143 & 140 & 1 & 1149.1 & 1165.4 \\
\hline 60 & 143 & 141 & 1 & 1160.0 & 1172.7 \\
\hline 60 & 143 & 142 & 1 & 1171.0 & 1181.7 \\
\hline 60 & 143 & 143 & 1 & 1182.0 & 1188.5 \\
\hline 60 & 143 & 144 & 1 & 1187.5 & 1197.1 \\
\hline 60 & 143 & 145 & 1 & 1193.0 & 1203.5 \\
\hline 60 & 143 & 146 & 1 & 1198.5 & 1211.6 \\
\hline 60 & 143 & 147 & 1 & 1203.9 & 1217.5 \\
\hline 60 & 143 & 148 & 1 & 1209.4 & 1225.2 \\
\hline 60 & 143 & 149 & 1 & 1214.8 & 1230.7 \\
\hline 60 & 143 & 150 & 1 & 1220.3 & 1237.9 \\
\hline 60 & 143 & 151 & 1 & 1225.7 & 1243.0 \\
\hline 60 & 143 & 152 & 1 & 1231.1 & 1249.9 \\
\hline 60 & 143 & 153 & 1 & 1236.5 & 1254.6 \\
\hline 60 & 143 & 154 & 1 & 1241.9 & 1261.0 \\
\hline 60 & 143 & 155 & 1 & 1247.2 & 1265.4 \\
\hline 60 & 143 & 156 & 1 & 1252.6 & 1271.5 \\
\hline 60 & 143 & 157 & 1 & 1257.9 & 1275.4 \\
\hline 60 & 143 & 158 & 1 & 1263.3 & 1281.2 \\
\hline 60 & 143 & 159 & 1 & 1268.6 & 1284.8 \\
\hline 60 & 143 & 160 & 1 & 1273.9 & 1290.2 \\
\hline 60 & 143 & 161 & 1 & 1279.2 & 1293.5 \\
\hline 60 & 143 & 162 & 1 & 1284.5 & 1298.5 \\
\hline 60 & 143 & 163 & 1 & 1289.8 & 1301.5 \\
\hline
\end{tabular}




\section{Conclusions}

It may be noted that, the two theories upon which all modern physics rests are general relativity (GR) and quantum field theory (QFT). GR is a theoretical framework that only focuses on the force of gravity for understanding the universe in regions of both large-scale and high-mass: stars, galaxies, clusters of galaxies, etc. On the other hand, QFT is a theoretical framework that only focuses on three non-gravitational forces for understanding the universe in regions of both small scale and low mass: sub-atomic particles, atomic nuclei, atoms, molecules, etc. QFT successfully implemented the Standard Model and unified the three non-gravitational interactions. But, so far no model succeeded in coupling and understanding the unified concepts of gravitational interaction and electromagnetic and strong interactions.

Qualitatively and quantitatively, from the above concepts and relations, the proposed three assumptions can be given some priority at fundamental level. With further research and analysis, basics of final unification can be explored. The authors are working on interconnecting the three gravitational constants in a unified manner and it will be submitted elsewhere very soon.

\section{Acknowledgements}

Author Seshavatharam U.V.S is indebted to professors K.V. Krishna Murthy, Chairman, Institute of Scientific Research in Vedas (I-SERVE), Hyderabad, India and Shri K.V.R.S. Murthy, former scientist IICT (CSIR), Govt. of India, Director, Research and Development, I-SERVE, for their valuable guidance and great support in developing this subject. Both of the authors are very much thankful to the anonymous reviewers for their valuable comments and kind suggestions in improving the quality of this paper.

\section{REFERENCES}

[1] G.W.Gibbons. The Maximum Tension Principle in General Relativity. Found.Phys.32:1891-1901 (2002)

[2] Roger Penrose. Chandrasekhar, Black Holes, and Singularities. J. Astrophys. Astr. 17, 213-231(1996)

[3] Subrahmanyan Chandrasekhar. On Stars, Their Evolution and Their Stability. Nobel Prize lecture, (1983)

[4] U. V. S. Seshavatharam, Lakshminarayana S. Final unification with Schwarzschild's Interaction. Journal of Applied Physical Science International 3(1): 12-22 (2015).

[5] U. V. S. Seshavatharam, Lakshminarayana S. Understanding Nuclear Structure With Final unification Journal of Applied Physical Science International 4(4): 191-295 (2015).

[6] U. V. S. Seshavatharam, Lakshminarayana S. To confirm the existence of nuclear gravitational constant, Open Science Journal of Modern Physics. 2(5): 89-102 (2015)

[7] U. V. S. Seshavatharam, Lakshminarayana S. Understanding Nuclear Stability, Binding Energy and Magic Numbers with Fermi Gas Model. Journal of Applied Physical Science International, 4 (2) pp.51-59 (2015)

[8] P.J. Mohr, B.N. Taylor, and D.B. Newell . CODATA Recommended Values of the Fundamental Physical Constants:2010" by in Rev. Mod. Phys. 84, 1527 (2012)

[9] K.A. Olive et al. (Particle Data Group), Chin. Phys. C, 38, 090001 (2014)

[10] Chowdhury, P.R. et al. Modified Bethe-Weizsacker mass formula with isotonic shift and new driplines. Mod. Phys. Lett. A20 p.1605-1618.(2005) 